\title{
Clinical profile of neonates delivered from mothers with confirmed COVID-19 infection:An experience from a Tertiary Perinatal Care Center in Dubai, UAE
}

\begin{abstract}
Background: The Coronavirus disease 2019 (COVID-19) outbreak is caused by the novel severe acute respiratory syndrome coronavirus 2 (SARS-CoV-2). ${ }^{1}$ On January 30 , 2020, the World Health Organization (WHO) declared the outbreak, as a Public Health Emergency of international concern and later on upgraded the outbreak on 11 March 2020, to a Pandemic. Neonates are a special population who might have different level of risks and susceptibilities to the virus. The risk of in-utero (vertical transmission) transmission of SARS-CoV-2 is anticipated to be low. The risk of perinatal transmission, especially during breastfeeding and the neonate's risk of developing COVID-19 during the perinatal period are also unknown. Until date, the knowledge is limited on whether rooming-in the neonate with mother (if she is SARS-COV-2 positive) after delivery is a safe practice.
\end{abstract}

Methods: This is a retrospective observational study, which is conducted at the Neonatal Intensive Care Unit (NICU) and post-natal wards of Latifa Women and Children Hospital (LWCH), Dubai, United Arab Emirates (UAE). We conducted this study aiming to evaluate and follow up neonates born to SARS-COV-2 positive mothers. In addition, to find out any potential risk factors associated with transmission of infection and elucidate best infection control and management practices. Maternal and neonatal data were collected retrospectively from our electronic medical records. Testing for neonates for SARS-COV-2 infection was done by real time reverse transcriptase Polymerase Chain Reaction (rtPCR) performed on nasopharyngeal swab samples. Samples are collected at birth (or as soon as possible) and 24-48 hours after the first sample; in case of any positive result, subsequent samples collected after 5-7days (at 24 hours interval), until two consecutive samples were negative. Universal COVID-19 screening for pregnant women presented for delivery was adopted.

Results: Between $1^{\text {st }}$ March 2020 and $15^{\text {th }}$ August 2020, a total of 92 pregnant women were admitted to our hospital and diagnosed positive for SARS- COv-2 infection. Out of which, 35 mothers and their 36 neonates (one was twin delivery) were included for analysis. Two (5.5\%) neonates were confirmed positive for SARS-COV-2 infection and one had inconclusive report. All neonates were stable and asymptomatic, and their subsequent rtPCR tests were negative. All the neonates (including the positive cases) were roomedin together with their mother and exclusive breast-feeding was given unless not feasible. These practices did not increase the risk of neonatal SARS-COV-2 infection. All neonates were discharge home in good condition. Neonates were clinic follow up (outpatient clinic or telephonic) and were found to be in healthy condition.

Conclusion: In our current study, we found out that transmission rate of SARS-COV-2 from mother to child is minimal if proper identification of infections and proper education to mother and adherence to infection control practice is ensured. If possible, mother and baby should be cared in a single room and exclusive breast-feeding practice could be feasible without any additional risk of transmission of SARS-COV-2 infection to the neonates.
Volume 10 Issue 5 - 2020

\author{
Mahmoud ElHalik,' Swarup Dash, ${ }^{2}$ Khaled \\ EL-Atawi, ${ }^{3}$ Rim Mahfouz, ${ }^{4}$ Ahmed Mabrouk, ${ }^{4}$ \\ Diana D'Souza, ${ }^{5}$ Kefah El Debek, ${ }^{6}$ Soosan \\ Varughese, ${ }^{6}$ Nancy Augustine ${ }^{6}$ \\ Consultant Neonatologist, Head of Neonatal Intensive Care \\ Unit, UAE \\ ${ }_{2}^{2}$ Senior Specialist, NICU, Pediatric Department, UAE \\ ${ }^{3}$ Consultant Neonatologist, NICU, Pediatric Department, UAE \\ ${ }^{4}$ Specialist Registrar, NICU, Pediatric Department, UAE \\ ${ }^{5}$ Nurse Supervisor, Nursing Department, UAE, \\ ${ }^{6}$ Charge Nurse, Nursing Department, UAE
}

Correspondence: Dr. Mahmoud Saleh Elhalik, Head of NICU, Latifa Women \& Children Hospital, DHA, Dubai, UAE, EmailmsElHalik@dha.gov.ae

Received: October II, 2020 | Published: November II, 2020

\section{Introduction}

The Coronavirus disease 2019 (COVID-19) outbreak is caused by the novel severe acute respiratory syndrome coronavirus 2 (SARSCoV-2). The disease first emerged in Wuhan province of China in early December 2019. ${ }^{1}$ The World Health organization (WHO) declared the outbreak a public health emergency of international concern on January30, 2020 and a pandemic on $11^{\text {th }}$ March 2020. ${ }^{2}$ The virus has rapidly spread causing a global pandemic with a major burden on the health care system and economy. Infecting millions of people and causing more than a million deaths [case fatality rate (CFR): $2.97 \%$, WHO Coronavirus Disease (COVID-19) Dashboard. ${ }^{3}$
Coronaviruses are single-strand, positive-sense RNA viruses with spike-like projections on their surface. These viruses can infect both animals and humans. ${ }^{4}$ Through the $\mathrm{S}$ protein (envelop spike protein) SARS-CoV-2 infects the host cells, it mediates the binding and membrane fusion through the angiotensin-converting enzyme 2 (ACE-2) receptor. ${ }^{5}$ Majority of ACE-2 receptors are found in alveolar epithelial type II cells in the lungs, although they are expressed in many tissues. By down regulating the ACE-2 intracellular signaling (mitochondrial assembly receptor), SARS-COV-2 causes inflammation, vasoconstriction, and fibrosis in the lung. ${ }^{6}$

The overall understanding is that this disease is more dangerous in adults with increasing age along with co-morbidities, compared 
to children. ${ }^{7}$ Few studies focused on understanding the clinical characteristics in the Neonates. Neonates are a special population who might have different level of risks and susceptibilities. ${ }^{7,8}$ They can be infected by intrauterine transmission, during delivery, from breast milk or from infected mother and family members in postnatal period. The risk of in-utero (vertical transmission) transmission of SARS$\mathrm{CoV}-2$ is anticipated to be low. ${ }^{9}$

Case reports describing isolation of SARS-CoV-2 from amniotic fluid and placental tissue and isolation of SARS-CoV-2 from the nasopharynx of neonates within $48 \mathrm{~h}$ of life suggested probable congenital infection. However, the rate of such infection in exposed neonates is unknown. ${ }^{10,11}$ The risk of perinatal transmission, especially when breastfeeding, and the neonate's risk of developing COVID-19 infection are also unknown. We are still not clear whether to roomingin the neonate with mother (if she is COVID-19 positive) after delivery. ${ }^{10,11}$ There are many recommendations globally regarding management of mother - infant dyad, but they differ on the management strategies due to scarcity of clinical data. ${ }^{12-16}$

This study was conducted aiming to evaluate and follow up the neonate born to SARS-COV-2 positive mother. We also aimed at finding out potential risk factors associated with transmission of infection and elucidate best infection control and management practices.

\section{Material and methods}

\section{Study design and population:}

This is a retrospective observational study, which is conducted at the Neonatal Intensive Care Unit (NICU) and post-natal wards of Latifa Women and Children's Hospital (LWCH), Dubai, United Arab Emirates (UAE). We retrieved all clinical information of the patients from their electronic medical records. We included all neonates delivered to mothers who tested positive for SARS-COV-2 infection, confirmed by real time reverse transcriptase polymerase chain reaction (rtPCR) performed on nasopharyngeal swab samples collected before delivery. The included neonates were born between $1^{\text {st }}$ March 2020 and $15^{\text {th }}$ August 2020. In our hospital we adopt universal COVID-19 screening for pregnant women presented for delivery; this is to prevent inadvertent exposure to healthcare providers and others from asymptomatic patients. This study was approved by Dubai Scientific Research Ethics Committee (DSREC), Dubai Health Authority (DSREC-07/2020_17).

\section{Procedures}

Diagnosis of COVID-19 infection was done by real time reverse transcriptase polymerase chain reaction (rtPCR) performed on nasopharyngeal swab samples. First sample was collected at birth (or as soon as possible) and $2^{\text {nd }}$ sample collected $24-48$ hours after the first sample; in case of any positive result, subsequent samples collected after 5-7days (at 24 hours interval) until two consecutive results were negative.

Universal COVID-19 screening for pregnant women was carried out. All necessary precautions (including wearing complete Personal Protective Equipment (PPE)), were taken by the health care providers who attended the delivery. Care of delivered neonate was as per our COVID-19 and latest neonatal resuscitation program (NRP) guideline. If mother and neonates were stable then both are roomedin a separate isolation room. Neonates were kept in a separate baby cot placed at least 6 feet away from mother. Mother was educated in infection prevention practices like; wearing mask, hand washing and precautions to be taken during caring for the baby and breastfeeding. Breastfeeding was encouraged, if not possible expressed breast milk was provided. Separation of mother and baby was done only for medical reasons. If neonates were transferred to neonatal intensive care unit (NICU) then they were kept in designated isolation rooms. Expressed breast milk (EBM) was provided if available. While neonates are in NICU, mother is allowed to visit the neonates only when she is free of symptoms and two consecutive nasopharyngeal rtPCR samples are negative. When mother and neonate are stable, they were either transfer to a designated Covid-19 facility or discharge home with proper education and regular follow-up ensured. Post discharge infants followed up either in outpatient clinic or through telephonic interview of parents

\section{Study objectives:}

Primary objectives: To find out the transmission risk of COVID-19 infection from mother to neonate.

\section{Secondary objectives:}

- Clinical profile of children born from COVID-19 positive mother.

- Feasibility of rooming-in and breast feeding practice.

- Condition of the baby on subsequent follow up.

\section{Data collections}

Data collected retrospectively from electronic medical records and includes; demographics, maternal and neonatal clinical presentation at time of delivery, during hospitalization and subsequent follow up. Neonates were accessed regularly for development of symptoms like; fever, hypothermia, lethargy, poor feeding, irritability, diarrhea, respiratory distress and other signs of respiratory infections. We also collected information related to infection control practices like; disposition of the neonate at delivery (allowed to room in with mother or admitted to a dedicated isolation room), feeding method (direct breastfeeding or expressed breast milk or formula) and other hygienic practices.

\section{Statistical analysis}

Descriptive analyses were used with results presented as proportions for categorical variables, median and interquartile ranges for continuous variables. Data were analyzed using Microsoft excel 2016 and online calculators.

\section{Results}

A total of 92 pregnant women were admitted to our hospital and diagnosed positive for SARS-COV-2 infection during the study period. Out of which, 35 mothers were positive during delivery, and total numbers of neonates born from them were 36 , as one was twin delivery and were included for analysis. Table 1 summarizes the characteristics of mothers. Average age of mothers was 32 years at the time of delivery. Majority of mothers $(31.4 \%, \mathrm{~N}=11)$ were UAE nationals. Twenty percent $(\mathrm{N}=7)$ were symptomatic with onset of symptom occurred in an average 6 days before delivery (Median 6 days and range 0-10days). Median time interval between symptoms onset and delivery was 4 days (range 0-10 days). About one-third ( $\mathrm{N}=13$, $37.1 \%$ ) of pregnancy was complicated by gestational diabetes and further $8.6 \%$ had Pregnancy induced hypertension $(\mathrm{N}=3)$. Premature rupture of membrane was present in two cases $(5.7 \%)$ and amniotic fluid was found to be meconium stained during two deliveries $(5.7 \%)$. One fourth (N 8) of the delivery were caesarean section. There was one maternal death due to COVID-19 related complications. 
Table I Maternal data: (with Perinatal COVIDI9 infection)

\begin{tabular}{|c|c|}
\hline \multicolumn{2}{|l|}{$\mathbf{N}=\mathbf{3 5}$} \\
\hline Age (years) & $32(24-42)$ \\
\hline \multicolumn{2}{|l|}{ Nationality: $*$} \\
\hline UAE & II (3I.4) \\
\hline India & $6(17.1)$ \\
\hline Pakistan & $5(14.3)$ \\
\hline Philippines & $5(14.3)$ \\
\hline Others & $10(28.6)$ \\
\hline Symptomatic before delivery $*$ & $7(20)$ \\
\hline Symptoms onset (days before delivery)\# & $6(0-10)$ \\
\hline COVID- 19 positive (days before delivery)\# & $4(0-10)$ \\
\hline \multicolumn{2}{|l|}{$\begin{array}{l}\text { Other medical conditions complicating } \\
\text { pregnancy:* }\end{array}$} \\
\hline $\mathrm{PIH}$ & $3(8.6)$ \\
\hline Gestational diabetes & I3 (37.I) \\
\hline PROM & $2(5.7)$ \\
\hline Chorioamnionitis & I (2.8) \\
\hline MSAF & $2(5.7)$ \\
\hline \multicolumn{2}{|l|}{ Mode of delivery:* } \\
\hline Vaginal & $9(25.7)$ \\
\hline LSCS & $27(74.3)$ \\
\hline Maternal death & I (2.8) \\
\hline
\end{tabular}

Infant characteristics are depicted in Table 2. A total of 36 neonates were included in the study; out of which 19 (52.8\%) were male and $17(47.2 \%)$ were female. Number of babies delivered preterm were $12(33.3 \%)$ and $24(66.7 \%)$ were term with median gestational age of 37 weeks (range 33-40, IQR 3). Median weight at birth was $2985 \mathrm{gms}$ (range 1500-3845gms). One fourth of neonates $(\mathrm{N}=9)$ were admitted to NICU. Prematurity was the major cause ( $\mathrm{N}=7 / 9,77.8 \%)$ of NICU admission. Among the babies who were admitted to NICU; 4 (44.4\%) had symptoms of respiratory distress and $1(11.1 \%)$ had fever. Out of the 36 neonates, $27(75 \%)$ were roomed-in with mother. Four $(14.8 \%)$ were separated from their mother due to maternal illness and were kept in a dedicated isolation room in the neonatal unit. Most of our neonates $(\mathrm{N}=32,88.9 \%)$ received either direct breast feeding with infection control precautions or expressed breast milk (if breast feeding not feasible due to maternal and neonatal causes). All of the neonates who were roomed-in with mother received direct breast feeding with proper infection control practice which has been taught to the mother. None of those babies were positive for SARS-COV-2 except one baby, whose first sample taken just after birth was positive but subsequent tests were negative. None of our babies had significant congenital anomalies.
Table 2 Neonatal data: (born from Mother with confirmed COVIDI9 infection)

\begin{tabular}{|c|c|}
\hline \multicolumn{2}{|l|}{$\mathbf{N}=36$} \\
\hline Gender:* & \\
\hline Male & $19(52.8)$ \\
\hline female & $17(47.2)$ \\
\hline Gestational age (weeks)\# & $37(33-40)-$ IQR 3 \\
\hline Number of preterm $(<37$ weeks $) *$ & $12(33.3)$ \\
\hline Birth weight\# & $2985(1500-3845)$ \\
\hline Singleton* & $34(94.5)$ \\
\hline Twin8 & I (2.8) \\
\hline AGA * & $36(100)$ \\
\hline SGA* & 0 \\
\hline LGA* & 0 \\
\hline APGAR score:\# & \\
\hline I minutes & $8(6-9)-I Q R 2$ \\
\hline 5 minutes & $9(8-10)-I Q R 0$ \\
\hline Positive COVID-19 test:* & \\
\hline Positive & $2(5.5)$ \\
\hline Inconclusive & I (2.8) \\
\hline Admitted in NICU:* & $9(25)$ \\
\hline Prematurity & $7(77.8)$ \\
\hline Respiratory distress & $4(44.4)$ \\
\hline Fever & I (II.I) \\
\hline Roomed in with Mother:* & $27(75)$ \\
\hline $\begin{array}{l}\text { Separated from Mother due to maternal } \\
\text { sickness* }\end{array}$ & $4(14.8)$ \\
\hline Received breast milk (breast feeding/EBM)* & $32(88.9)$ \\
\hline Significant congenital anomalies* & 0 \\
\hline Discharge Home* & $36(100)$ \\
\hline Death * & 0 \\
\hline Neonates followed up & $25(69.4)$ \\
\hline
\end{tabular}




\begin{tabular}{|l|l|}
\hline What is known about this topic? & $\begin{array}{l}\text { - Low risk of vertical transmission of SARS-COV-2 infection from mother to baby. } \\
\text { - Uncertainty about care of stable neonates after delivery. }\end{array}$ \\
\hline What our study adds? & $\begin{array}{l}\text { - Vertical transmission of infection may be possible. } \\
\text { - There is no relation between symptoms of disease in mother and risk of trans- } \\
\text { mission. }\end{array}$ \\
$\begin{array}{l}\text { - If adequate infection control measure is undertaken the neonate could be safely } \\
\text { roomed in with mother and receive exclusive breast feeding and/or expressed } \\
\text { breast milk. }\end{array}$
\end{tabular}

Nasopharyngeal sample for rtPCR were collected for detection of infection as per our hospital protocol and the result for all are available. From which, two $(5.5 \%)$ were confirmed positive for SARS-COV-2 infection and one baby had inconclusive report. The first was a late preterm (36 weeks gestation) female neonate delivered by cesarean section with birth weight of $2460 \mathrm{gms}$. Her mother was positive one day before delivery and was asymptomatic. Her first nasopharyngeal rtPCR sample collected at birth was positive for SARS-COV-2. Two Subsequent samples taken after 5 days of positive results, 24 hours apart were negative. She was asymptomatic and was roomed-in with the mother. Direct breast-feeding was provided (with proper infection control precautions). The $2^{\text {nd }}$ neonate was a term, $3845 \mathrm{gms}$ birth weight, female and was delivered vaginally. Her mother was SARSCOV-2 positive 10 days prior, remained positive until delivery, and was having mild respiratory symptoms. First rtPCR sample collected at birth from $2^{\text {nd }}$ neonate was negative but $2^{\text {nd }}$ nasopharyngeal sample taken after 24 hours was positive. Two Subsequent samples taken after 5 days of positive results, 24 hours apart were negative. The $2^{\text {nd }}$ neonate was asymptomatic, remained with the mother, and received direct breast-feeding in a dedicated isolation room. The third neonates, his $2^{\text {nd }}$ nasopharyngeal sample collected after 24 hours of birth was inconclusive but subsequent tests showed negative results. He was a preterm 35 weeks neonate delivered by cesarean section due to maternal condition. He was admitted to NICU for respiratory distress and received noninvasive ventilator support for 2 days. He did not receive breast milk, as mother was sick and admitted in ICU. All the neonates were discharge home in good condition. Early outpatient clinic follow up was given for all the babies. Twenty-five out of the 36 neonates could be followed up post-discharge (outpatient follow up or telephonic interview with parents), including the neonates who were positive for SARS-COV-2 rtPCR. All were found to be in healthy condition.

\section{Discussion}

We evaluated the outcome of neonates born to SARS-COV-2 positive mothers. We also tried to find out the relation between maternal characteristics, caring mother-baby as a unit with roomingin and breast feeding and its effect on the neonates. To the extent of our knowledge; this is the largest cohort of neonates born to mothers who tested SARS-COV-2 positive at the time of delivery in Dubai and were followed up subsequently with serial testing and clinically when admitted in hospital and outpatient follow up after discharge.

Two $(5.5 \%)$ neonates were tested positive and both results were obtained within 48 hours of life. Vertical transmission risk could not be ruled out. There are several case reports available to date, of neonates who have tested positive for SARS-CoV-2 within $48 \mathrm{~h}$ of life. Zeng et.al., reported out of 33 neonates delivered from SARSCOV-2 positive mothers, three were positive with nasopharyngeal and anal swabs taken on days of life 2 and $4 .{ }^{17}$ Sheth et.al., in a narrative review of literature found that 10 out of 23 COVID-19 positive neonates have a chance of vertical transmission, they also postulated that, approximately $3 \%$ of the babies born to COVID-19 positive mother have some chances of vertical transmission. ${ }^{18}$ Few other reports also predicted possibility of vertical transmission but the overall risk for infant's remains to be determined. ${ }^{19,20}$ The low risk of vertical transmission has been hypothesized to be due to paucity of ACE2 receptors in the placenta, which may be necessary for transplacental transfer to the fetus. ${ }^{21}$

Our hospital is a mother and baby friendly hospital and we encourage direct breast-feeding and care of mother and baby as a unit. We did not observe a relation between this practice and risk of neonatal SARS-COV-2 infection. The recommendation worldwide varies greatly regarding this subject. There is paucity of evidence suggesting presence of SARS-COV-2 virus in breast milk. ${ }^{15,16,22-26}$ Breast milk samples from six infected mothers were tested negative for SARS-CoV-2 by Chen et al, but more information is needed to rule out this route of transmission. ${ }^{27}$ In the absence of any uniform recommendations and potential risk of transmission from roomingin and breast milk feeding; we recommend if adequate infection control precautions is followed then mother (even if they have mild symptoms) and baby should be roomed-in together and exclusive breast feeding practice should be followed. This will lead to mother infant bonding and transfer of benefit of breast milk to the neonates. This ultimately leads to better transition and sustenance of exclusive breast-feeding when discharged home.

Both the SARS-COVID-2 positive neonates in our study are female but our sample size is too small to delineate any gender difference of infection rate if there is any. We screened all pregnant women presented for delivery; as evidence suggested that asymptomatic carriers are common, which is confirmed in our cohort of mothers, with only $20 \%$ were symptomatic at the time of delivery. ${ }^{28}$

Strength of the study; we follow universal SARS-COV-2 screening practice of pregnant mothers admitted to our hospital, which could have prevented missing asymptomatic cases. This also leads to proper planning in care of mother and baby. Ours is one of the largest cohorts of neonates born to mothers who tested positive for SARS-CoV-2 at time of delivery and subsequently followed up. We tried to solve the riddle behind care of infected mother and their baby as a unit and breast feeding and concluded that such practice can be followed if adequate precautions are taken.

We also have few limitations; observational study with retrospective collection of data. We were unable to screen for presence of the virus in other samples like breast milk, amniotic fluids, blood, urine, or stool. Our sample size is limited which could have prevented us from finding any minimal differences in maternal and infant characteristics which might have impact on infection rate and outcome for which a large prospective study is advised.

\section{Conclusion}

Transmission rate of SARS-COV-2 from mother to child is minimal if proper identification of infections and strict infection control precautions are taken. If possible, mother and baby should 
be cared for in a single room and exclusive breast feeding practice should be followed. Most of the SARS-COV-2 positive neonates were asymptomatic. Implementation of uniform guideline and close collaborations between all departments in the hospital could help us tide over this difficult time. However, many facts regarding this infection, its course and management remained unanswered and a large prospective trial is advised.

\section{Conflicts of interest}

None.

\section{Funding}

None.

\section{References}

1. Zhu N, Zhang D, Wang W, et al. A novel coronavirus from patients with pneumonia in China, 2019. N Engl J Med. 2020;382(8):727-733.

2. WHO. Coronavirus disease 2019 (COVID-19).

3. WHO Coronavirus Disease (COVID-19) Dashboard.

4. Zimmerman P, Curtis N. Coronavirus infections in children including COVID- 19: an overview of the epidemiology, clinical features, diagnosis, treatment and prevention options in children. Pediatr Infect Dis J. 2020;39(5):355-368.

5. Fu Y, Cheng Y, Wu Y. Understanding SARS-CoV-2-mediated inflammatory responses: from mechanisms to potential therapeutic tools. Virol Si. 35(3):266-271.

6. Zhang H, Penninger JM, Li Y, et al. Angiotensin-converting enzyme 2 (ACE2) as a SARS-CoV-2 receptor: molecular mechanisms and potential therapeutic target. Intensive Care Med. 46(4):586-590.

7. Dong Y, Mo X, Hu Y, et al. Epidemiology of COVID-19 among children in China. Pediatrics. 2020;145(6):e20200702.

8. Lu X, Zhang L, Du H, et al. SARS-CoV-2 infection in children. $N$ Engl J Med. 2020;382(17):1663-1665.

9. Zamaniyan M, Ebadi A, Aghajanpoor Mir S, et al. Preterm delivery in pregnant woman with critical COVID-19 pneumonia and vertical transmission. Prenat Diagn. 2020.

10. Kirtsman M, Diambomba Y, Poutanen SM, et al. Probable congenital SARS-CoV-2 infection in a neonate born to a woman with active SARSCoV-2 infection. CMAJ. 2020.

11. Zhu H, Wang L, Fang C, et al. Clinical analysis of 10 neonates born to mothers with 2019-nCoV pneumonia. TranslPediatr. 2020;9:51-60.

12. Chen H, Guo J, Wang C, et al. Clinical characteristics and intrauterine vertical transmission potential of COVID-19 infection in nine pregnant women: a retrospective review of medical records. Lancet. 2020; 395 : 809-815.
13. American Academy of Pediatrics. FAQs: Management of infants born to mothers with COVID-19. American Academy of Pediatrics, 2020.

14. Wang L, Shi Y, Xiao T, et al. Chinese expert consensus on the perinatal and neonatal management for the prevention and control of the 2019 novel coronavirus infection (First edition). Ann Transl Med. 2020;8:47.

15. WHO. Clinical management of COVID-19. Interim guidance. World Health Organization, May 27, 2020.

16. CDC. Evaluation and management considerations for neonates at risk for COVID-19. Centers for Disease Control and Prevention, 2020.

17. Zeng L, Xia S, Yuan W, et al. Neonatal early-onset infection with SARSCoV-2 in 33 neonates born to mothers with COVID-19 in Wuhan, China. JAMA Pediatr. 2020;174(7):722-725.

18. SudipSheth, Nidhi Shah, Vineet Bhandari. Outcomes in COVID-19 Positive Neonates and Possibility of Viral Vertical Transmission: A Narrative Review. Am J Perinatol. 2020;37(12):1208-1216.

19. Zamaniyan M, Ebadi A, Aghajanpoor Mir S, et al. Preterm delivery in pregnant woman with critical COVID-19 pneumonia and vertical transmission. Prenat Diagn. 2020;10.1002/pd.5713.

20. Knight M, Bunch K, Vousden N, et al. Characteristics and outcomes of pregnant women admitted to hospital with confirmed SARSCoV-2 infection in UK: national population based cohort study. BMJ . 2020;369:2107.

21. Egloff C, Vauloup-Fellous C, Picone O, et al. Evidence and possible mechanisms of rare maternal-fetal transmission of SARS-CoV- $J$ ClinVirol. 2020;128:104447.

22. Royal College of Paediatrics and child Health. COVID-19 - guidance for neonatal settings, 2020.

23. American Academy of Pediatrics. FAQs: Management of infants born to mothers with COVID-19. American Academy of Pediatrics, 2020.

24. Davanzo R, Moro G, Sandri F, et al. Breastfeeding and coronavirus disease-2019: ad interim indications of the Italian Society of Neonatology endorsed by the Union of European Neonatal \& Perinatal Societies. Matern Child Nutr. 2020;16(3):e13010.

25. American Academy of Pediatrics. FAQs: Management of infants born to mothers with COVID-19. American Academy of Pediatrics, 2020.

26. Wang L, Shi Y, Xiao T, et al. Chinese expert consensus on the perinatal and neonatal management for the prevention and control of the 2019 novel coronavirus infection (First edition). Ann Transl Med. 2020; $8(3): 47$.

27. Chen H, Guo J, Wang C, et al. Clinical characteristics and intrauterine vertical transmission potential of COVID-19 infection in nine pregnant women: a retrospective review of medical records. Lancet. 2020;395(102226):809-815.

28. Wang Y, Liu Y, Liu L, et al. Clinical outcome of 55 asymptomatic cases at the time of hospital admission infected with SARS-Coronavirus-2 in Shenzhen, China. J Infect Dis. 2020; 221(11):1770-1774. 